\title{
Sentinel lesions of primary CNS lymphoma
}

\author{
L Alderson, M R Fetell, M Sisti, F Hochberg, M Cohen, D N Louis
}

Departments of

Neurology and

Neurosurgery,

Columbia

Presbyterian Medical

Center, New York, NY,

USA,

Neurology and

Neurosurgical Services

and Department of

Pathology

(Neuropathology),

Massachusetts General

Hospital and Harvard

Medical School,

Boston, MA, USA

and

Department of

Neuropathology,

University Hospitals of

Cleveland, Cleveland,

OH, USA

L Alderson

M R Fetell

$M$ Sisti

F Hochberg

$M$ Cohen

D N Louis

Correspondence to

Dr Michael R Fetell,

Neuro-Oncology Unit,

Neurological Institute,

Columbia-Presbyterian

Medical Center, $710 \mathrm{~W}$

168th Street, NY, NY

10032, USA.

Received 15 March 1995

and in revised form

10 August 1995

Accepted 7 September 1995

\begin{abstract}
Some patients ultimately diagnosed with primary CNS lymphoma (PCNSL) have transient symptomatic contrast enhancing lesions. These "sentinel lesions" of PCNSL recede spontaneously or with corticosteroid treatment and present an important diagnostic dilemma because they show variable, but non-diagnostic histopathological features.

Four previously healthy, immunocompetent patients aged 49 to 58 years had contrast enhancing intraparenchymal brain lesions. Before biopsy, three of the four were treated with corticosteroids. Initial biopsies showed demyelination with axonal sparing in two, non-specific inflammation in one, and normal brain in one. Infiltrating lymphocytes predominantly expressed $T$ cell markers with rare B cells. All four patients recovered within two to four weeks after the initial biopsy and imaging studies showed resolution of the lesions. The CSF was normal in three of the four patients tested; oligoclonal bands were absent in both of the two tested.
\end{abstract}

After seven to 11 months, each patient developed new symptomatic lesions in a different region of the brain, biopsy of which showed a B cell PCNSL. The mechanism of spontaneous involution of sentinal lesions is not understood, but may represent host immunity against the tumour. Sentinel lesions of PCNSL should be considered in patients with contrast enhancing focal parenchymal lesions that show non-specific or demyelinative histopathological changes. Close clinical and radiographic follow up is essential if PCNSL is to be diagnosed early in such patients.

(F Neurol Neurosurg Psychiatry 1996;60:102-105)

Keywords: primary CNS lymphoma; diagnosis; tumour regression; multiple sclerosis

Primary CNS lymphoma (PCNSL) is an uncommon neoplasm, representing about $1 \%$ of all intracranial tumours. However, for unknown reasons the incidence of PCNSL is increasing, even among immunocompetent people.' Before diagnosis of PCNSL some patients have enhancing intra-axial CNS lesions that regress spontaneously ${ }^{23}$ or with corticosteroid treatment. ${ }^{45}$ Therefore, PCNSL may simulate multiple sclerosis with waxing and waning brain lesions. ${ }^{6}$ We introduce the term "sentinel lesions" for the contrast enhancing focal lesions that precede by several months the histopathological diagnosis of PCNSL. We describe the clinical and histopathological findings in four patients who presented with sentinel lesions of PCNSL.

\section{Patient 1}

A 58 year old woman first noted intermittent diplopia in July 1989 that lasted four weeks and resolved spontaneously. In September 1989 she became progressively fatigued and somnolent over two weeks. Examination in September 1989 showed disorientation and weakness of the left leg. Brain CT and MRI disclosed an enhancing lesion with mass effect in the right hypothalamus. The CSF was normal and serum sodium content was low, consistent with inappropriate antidiuretic hormone secretion. Dexamethasone was started and she improved over the next two weeks. In November 1989, a CT guided stereotactic biopsy of the hypothalamic lesion showed a chronic inflammatory infiltrate and demyelination with axonal sparing. No intranuclear or cytoplasmic inclusions were seen and immunostaining for toxoplasmosis was negative. Immunostaining for L26 and Leu 22 antigens disclosed perivascular $\mathrm{T}$ cells with few B cells. A diagnosis of probable multiple sclerosis was made. The patient was discharged without symptoms and the corticosteroid dosage was reduced over the next four weeks.

Seven months later she again noted fatigue, disorientation, left sided weakness, and visual impairment. Brain CT showed enhancing lesions in the septum pellucidum, head of the right caudate, splenium of the corpus callosum, and at multiple sites in the periventricular white matter. The prior biopsy site in the hypothalamus appeared as a small lucency without enhancement. A stereotactic biopsy of the right caudate lesion in July 1990 showed a B cell lymphoma of the diffuse large cell subtype. 
Figure 1 Contrast enhanced MRI of patient 3. (A) April 1992; left basal ganglionic tumour. (B) October 1992; MR showing resolution of left basal ganglionic lesion. Neurological examination was normal at this time. (C) December 1992; right basal ganglionic mass is present, six weeks after scan shown in (B).

\section{Patient 2}

A 49 year old woman noted ataxia, vertigo, and deafness in her right ear for two weeks in December 1988. Examination showed ataxia, dysarthria, hearing loss in the right ear, nystagmus, and right facial weakness involving the frontalis muscle. A contrast enhancing mass in
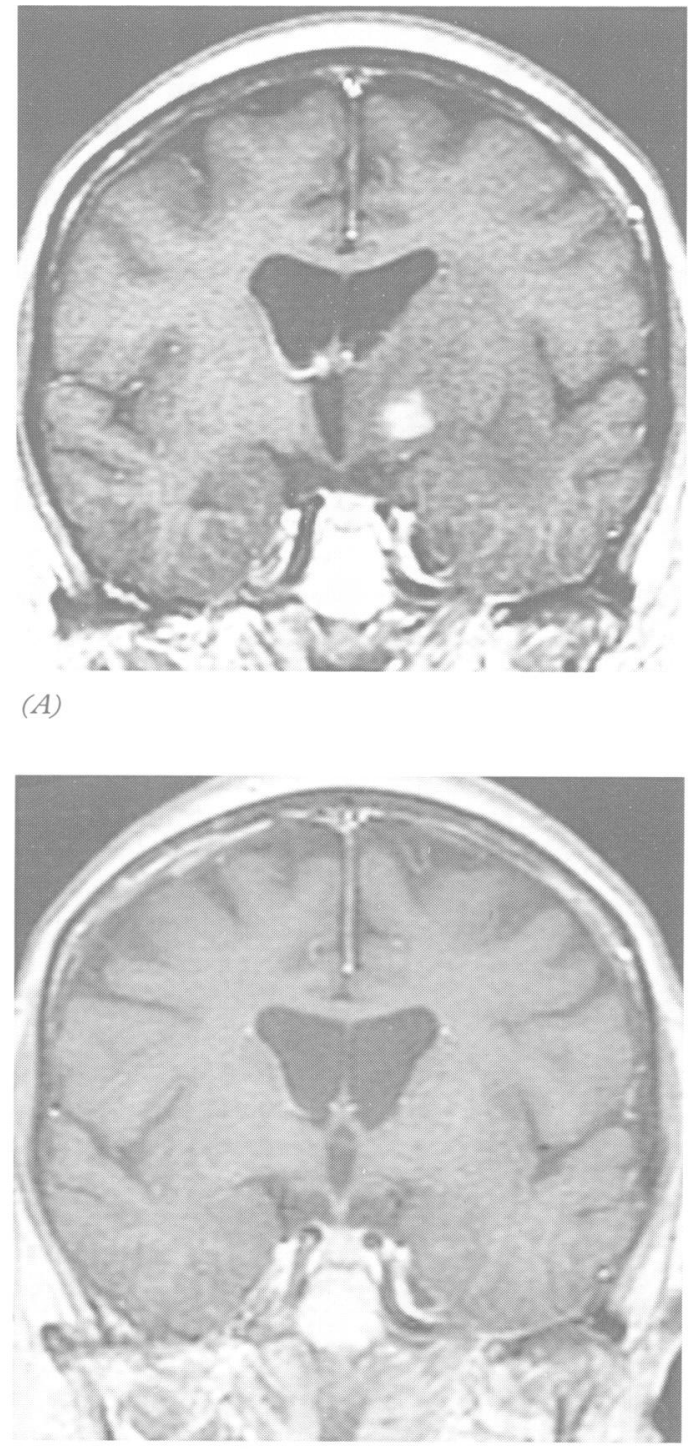

(B)

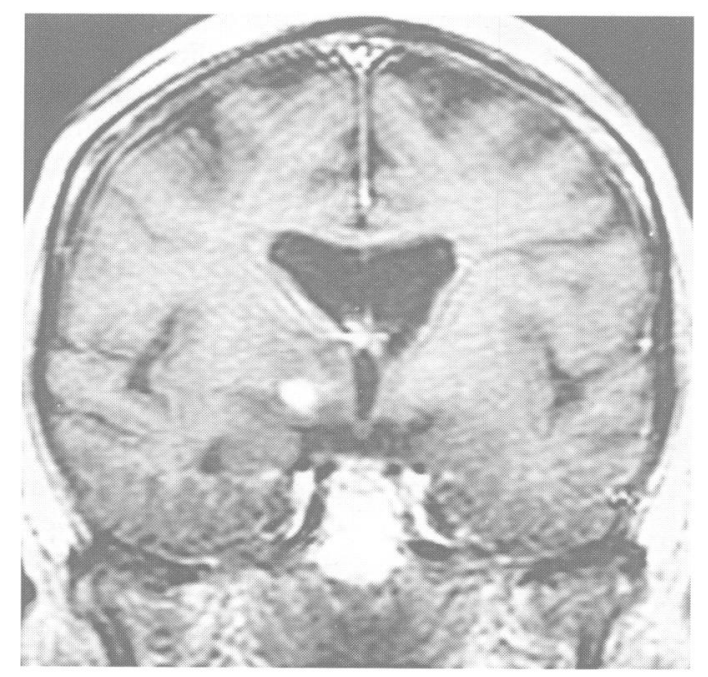

(C) the right lateral pons extending to the fourth ventricle was seen on brain CT and MRI. Suboccipital craniotomy and biopsy performed 10 days later showed a normal brain stem and cerebellar tissue with minimal inflammation. A surgical clip and postoperative scan confirmed that the biopsy came from the lesion, although it remains possible that the histological sample did not represent all abnormal areas. The patient was treated with corticosteroids for three weeks and her symptoms resolved. She was readmitted in March 1989 with vertigo and nystagmus. The previous lesion had completely resolved radiographically and CSF cytology was negative. She again improved with corticosteroid treatment.

In November 1989, 11 months after her initial symptoms, she had five weeks of progressive headache, confusion, and dysarthria. A contrast enhancing lesion was seen in the right frontal lobe on CT and MRI. A CT guided biopsy disclosed a B cell lymphoma of the diffuse large cell subtype. Serology for HIV was negative.

\section{Patient 3}

A 54 year old woman noted headache, right arm weakness, and dysarthria in April 1992. Brain CT and MRI showed an enhancing lesion in the left internal capsule (fig 1A) and she was started on phenytoin and dexamethasone. Serology for HIV was negative. Stereotactic biopsy of the involved white matter performed one week later showed perivascular inflammatory cells and hypertrophic astrocytes. Immunostaining with Leu22, UCHL1, $4 \mathrm{~KB} 5, \mathrm{~L} 26$, and $\mathrm{S} 100$ showed mostly $\mathrm{T}$ cells and macrophages with rare $B$ cells. She improved clinically over the following next two weeks and the medications were discontinued. Brain MRI in May 1992 showed resolution of the lesion (fig 1B) and neurological examination was normal. In January 1993 she developed a left hemiparesis and MRI showed a right internal capsule lesion with small satellite lesions in the periventricular white matter (fig 1C). Biopsy of the new lesion disclosed a malignant B cell lymphoma of the diffuse large cell subtype.

\section{Patient 4}

This 57 year old woman developed dysarthria and numbness in her right face and hand in November 1987. A contrast enhancing mass in the left parietal lobe was evident on CT and MRI showed this lesion as well as an area of increased signal on T2 in the right occipital lobe. She was treated with dexamethasone (6 $\mathrm{mg} /$ day) for two weeks before admission. Biopsy of the parietal lesion showed destruction of myelin with axonal sparing, many $T$ lymphocytes and foamy macrophages, rare $\mathrm{B}$ cells, and no evidence of neoplasm (fig 2). A diagnosis of probable multiple sclerosis was made and she was treated with a brief course of corticosteroids. Her symptoms resolved over two weeks and CT in April 1988 was 

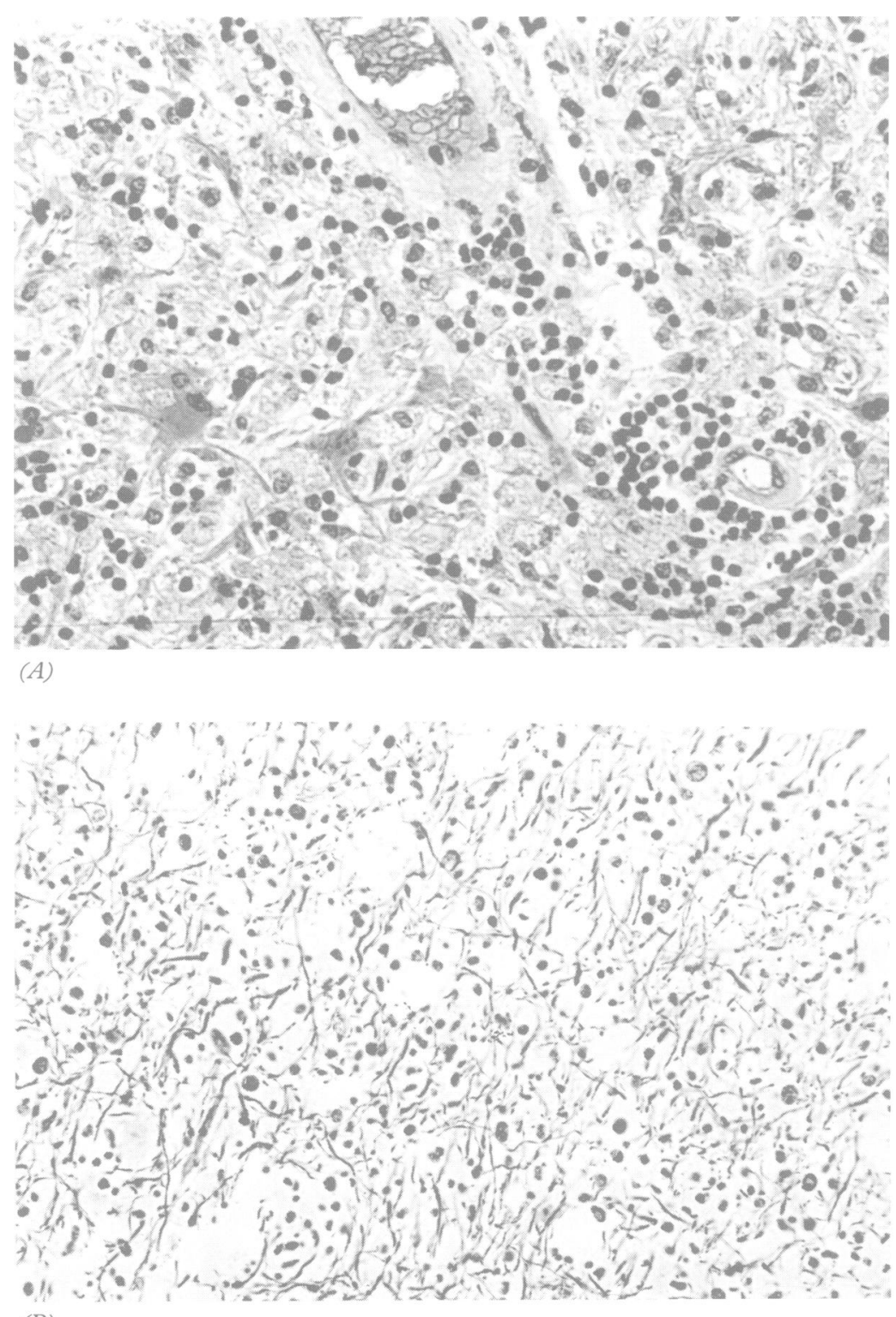

(B)

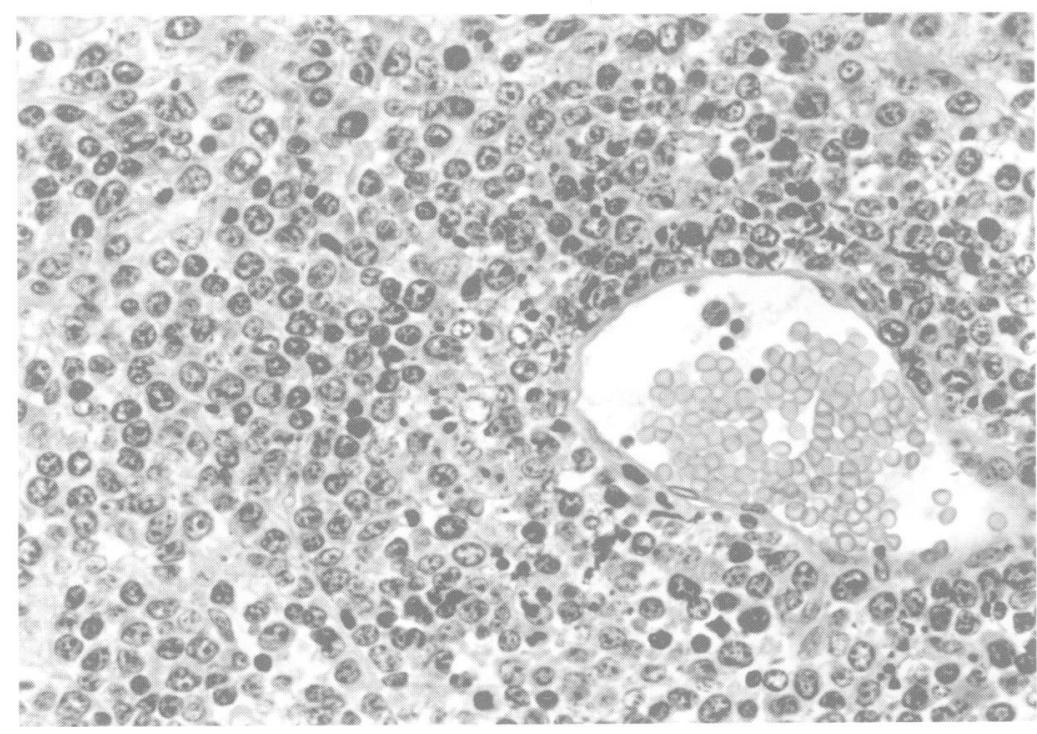

(C)

Figure 2 Photomicrographs of brain biopsies from patient 4. (A) Biopsy of the left parietal white matter shows reactive astrocytes, lymphocytes, and scattered macrophages containing luxol fast blue positive material consistent with myelin debris (luxol fast blue with haematoxylin and eosin, $\times 500)$. (B) Same biopsy as in $(A)$ with bodian silver impregnation showing axonal sparing $(\times 250)$. (C) Biopsy of the right parietal white matter nine months later showing perivascular sheets of large, mitotically active lymphoid cells characteristic of large cell lymphoma (haematoxylin and eosin $\times 500$ ). normal. In August 1988 she developed left hemisensory loss and CT showed a large, homogeneous, right parietal contrast enhancing mass. Biopsy showed a B cell lymphoma of the immunoblastic type (fig $2 \mathrm{C}$ ). This patient was included in a report of a series of patients with CNS demyelinating disease of unknown cause (patient 14, Kepes ${ }^{7}$ ).

\section{Discussion}

Occasionally, the diagnosis of PCNSL is missed because the initial lesion regresses spontaneously or after corticosteroid treatment. Even biopsy of the suspected tumour may be non-diagnostic. In the four immunocompetent patients presented here, the initial enhancing lesion was thought to be a brain tumour, but a biopsy showed unexpected findings. In one the brain appeared normal, in another non-specific inflammation was present, and in two patients demyelination with axonal sparing was seen. A normal brain biopsy might imply that tissue adjacent to the region of true pathology was sampled, but normal tissue was seen in patient 2 in whom a surgical clip was placed in the area of the resection, confirming the complete removal of the lesion.

Corticosteroids are cytotoxic to lymphocytes and may have contributed to tumour regression before biopsy in patients 1,3 , and 4. Patient 2, however, did not receive corticosteroids before her first biopsy, and resembles other cases of spontaneous radiographic ${ }^{3}$ and histological $^{4}$ tumour regression. Tumour regression in PCNSL is probably not simply due to the effects of exogenous corticosteroids.

The lymphocytes infiltrating the lesions expressed $\mathrm{T}$ lymphocyte markers, with only rare $\mathrm{B}$ cells, supporting the suspicion that inflammation plays a part in the resolution of these lesions. Infiltrates of $\mathrm{T}$ cells in $\mathrm{B}$ cell PCNSL lesions have been described," and probably reflect a cell mediated immune response mounted against their lymphoma by immunocompetent people. Likewise, when drug induced immunosuppression is reduced in organ transplant patients with lymphoma, the tumour may recede without specific antitumour therapy.

Demyelinating disease is probably the major differential diagnostic consideration in patients with sentinel lesions of PCNSL. Both PCNSL and demyelinating disease affect the white matter, and, as shown here, may have a remitting course. Acute demyelinating lesions may show contrast enhancement on MRI, mimicking a tumour. Two of our patients were thought to have multiple sclerosis or another demyelinating disease based on the biopsy report of demyelination with axonal sparing. In fact, as mentioned earlier after her first biopsy, patient 4 was reported as part of a series of cases of demyelinating disease. Her subsequent course was typical for a sentinel lesion; after a lag of nine months a new lesion appeared in another brain location and was biopsied with the finding of PCNSL. By contrast with typical patients with multiple 
sclerosis, the patients in our series are older and did not have prior clinical or radiographic lesions.

In rare instances both multiple sclerosis and PCNSL may occur in the same patient. Bender et $a l^{9}$ reported a 20 year old woman who had three prior episodes of neurological deficits that resolved with corticosteroids and a brain biopsy showing demyelinating disease eight years before the diagnosis of PCNSL. Necropsy disclosed only two small foci of demyelination and diffuse cerebral involvement with PCNSL. The eight year lag between diagnoses implies that the patient had both multiple sclerosis and PCNSL.

In a large neuropathological study of focal demyelinating lesions, Kepes suggested that intermittent immunosuppressive treatment could contribute to the development of PCNSL. ${ }^{7}$ However, if PCNSL was induced by the immunosuppression in our patients, an alternative aetiology would be required to explain the first lesions. Immunosuppressed patients display a lag time to development of lymphoma inversely proportional to the intensity of the immunosuppression. For transplant patients the average lag to appearance of lymphoma was 48 months for patients treated with cyclophosphamide-azathioprine. ${ }^{10}$ This is much longer than the seven to 11 month intervals seen in our patients. In addition, corticosteroid treatment in our patients was brief, lasting for weeks or a few months. Therefore, it seems unlikely that corticosteroids fostered the development of PCNSL.

Despite the varied pathological appearance of the lesions, the clinical syndrome of sentinel lesions is remarkably consistent. All patients had contrast enhancing lesions that looked like brain tumours, but regressed either with corticosteroids or spontaneously. Our experience with the first patients with this syndrome led us to tell patient 3 after her initial non-diagnostic biopsy that we were concerned about the diagnosis of PCNSL. We advised close clinical and radiographic follow up. Despite careful surveillance for recurrence, when the second focus of tumour appeared, it progressed rapidly, and a severe hemiparesis developed within days. This explosive course suggests reactivation of disease in another site, rather than an indolent lesion detected when it reached a critical size.

The seven to 11 month interval between sentinel and diagnostic biopsies in these four patients was relatively consistent. It is possible that lymphoma is kept dormant by host immunity. In a mouse model of systemic lymphoma, a prolonged dormant period can be induced with passive immunity to the tumorigenic $B$ cells. ${ }^{11}$ In molecular analysis of recurrent systemic B cell tumours, the immunoglobulin genes in a neoplastic clone continuously undergo mutation, eventually escaping autoimmune tumour destruction. ${ }^{12}$ PCNSL may be a multifocal disease from the onset, and the latency between the first and second tumours could be the time taken for another neoplastic clone to emerge.

It is important to stress the fact that the diagnosis of "sentinel lesions" can be suspected on clinical grounds. When a patient 50 years or older has a contrast enhancing focal lesion, even if the biopsy shows demyelinating changes, the diagnosis of PCNSL should be seriously considered. This should lead to changes in management including early discontinuation of corticosteroids, repeat biopsy (in certain instances), and close clinical and radiographic surveillance of the patient. The main differential diagnosis is multiple sclerosis which is often treated with long term corticosteroid therapy or immunosuppressive therapy that would likely obscure or delay the correct diagnosis. Admittedly, even with careful monitoring, patients do develop recurrent tumour that may be resistant to therapy; however, early diagnosis and treatment will still afford the best prognosis.

1 Eby NL, Gruffman S, Flannelly CM, Schold SC, Voge FS, Burger PC. Increasing frequency of primary brain lymphoma in the US. Cancer $1988 ; 62: 2461-5$.

2 Weingarten KL, Zimmerman RD, Leeds NE. Spontaneous regression of intracerebral lymphoma. Radiology $1983 ; 149 \cdot 721-4$

3 Williams RS, Crowell RM, Fisher CM, et al. Clinical and radiologic remission in reticulum cell sarcoma of the brain. Arch Neurol 1979;36:206-10.

4 Vaquero J, Martinez R, Rossi E, Lopez R. Primary cerebra lymphoma: the ghost tumor. $\mathcal{F}$ Neurosurg 1984;60:174-6.

5 Kuroda Y, Kawasaki T, Haraoka S, et al. Autopsy report of primary CNS B-cell lymphoma indistinguishable from multiple sclerosis: diagnosis with immunoglobulin gene rearrangements analysis. F Neurol Sci 1992;111:173-9.

6 DeAngelis LM. Primary central nervous system lymphom imitates multiple sclerosis. 7 Neurooncol 1990;9:177-81.

7 Kepes JJ. brain. Large focal tumor-like demyelinating lesions of brain: Intermediate entity between multiple sclerosis and acute disseminated encephalomyelitis? A study of 31
patients. Ann Neurol 1993;33:18-27. patients. Ann Neurol 1993,33:18-27.

tarzl TE, Porter KA, Iwatsuki S, et al. Reversibility of lymphomas and lymphoproliferative lesions developing under cyclosporin-steroid therapy Lancet 1984;i:583-7.

9 Bender GP, Schapiro RT. Primary CNS lymphoma presenting as multiple sclerosis. Minn Med 1989;72:157-60

10 Penn I. Cancers complicating organ transplantation $N$ Engl f Med 1990;323:1767-9.

11 Yefenof E, Picker LJ, Scheuermann RH, Tucker TF Vitetta ES, Uhr JW. Cancer dormancy: isolation and characterization of dormant lymphoma cells. Proc Nat Acad Sci USA 1993;90:1829-33.

12 Meeker T, Lowder JN, Cleary ML, et al. Emergence of idiotype variants during therapy of $B$ cell lymphoma with anti-idiotype antibodies. $N$ Engl $f$ Med 1985;312: 1658-63. 